well as dogged in our efforts to build trusting and trustworthy alliances among scientists, publics, and those engaged in governance. This is anything but a blank check to scientists. This is a call to sincere engagement and fundamental cultural shift.

Perhaps I, like others, am seeing what I want to see in the Nuffield report, but this world could do with a bit more solidarity.

\section{References}

1. Mathews DJ, Chan S, Donovan PJ, et al. CRISPR: a path through the thicket. Nature 2015;527:159-161. DOI: 10.1038/527159a.
2. National Academies of Sciences, Engineering, and Medicine. Human Genome Editing: Science, Ethics, and Governance, Washington, DC: The National Academies Press, 2017.

3. Nuffield Council on Bioethics. Genome Editing and Human Reproduction: Social and Ethical Issues. London: Nuffield Council on Bioethics, 2018.

4. Brokowski C. Do CRISPR germline ethics statements cut it? CRISPR J 2018;1:115-125. DOI: 10.1089/crispr.2017.0024.

5. OECD. Inequality. Available online at www.oecd.org/social/inequality.htm (accessed July 26, 2018).

6. Burall S. Rethink public engagement for gene editing. Nature 2018;555:438-439. DOI: 10.1038/d41586-018-03269-3.

7. Hurlbut JB, Jasanoff $S$, Saha $K$, et al. Building capacity for a global genome editing observatory: conceptual challenges. Trends Biotech 2018;36: 639-641. DOI: 10.1016/j.tibtech.2018.04.009.

8. Montoliu L, Merchant J, Hirsch F, et al. ARRIGE arrives: toward the responsible use of genome editing. CRISPR J 2018;1:128-130. DOI: 10.1089/crispr.2018.29012.mon.

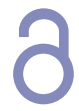

\title{
CRISPR-Cas9-Mediated Mutagenesis: Mind the Gap?
}

\author{
Lydia Teboul $^{1}$ and Andy Greenfield ${ }^{2}$
}

\section{A New Study Documenting CRISPR-Induced On-Target Large(r) Deletions and Rearrangements Highlights the Risks Associated with Such Events}

CRISPR*-Cas9 genome editing has created much excitement and no small amount of controversy. Previous alerts concerning its efficacy and safety included a report of widespread off-target effects. Following intense scrutiny, the design of that study proved to be flawed, the original article (published in Nature Methods) was retracted ${ }^{1}$ and the technology largely cleared of suspicion-potential off-target sequence changes were less frequent than those attributed to de novo mutation.

The most recent challenge to CRISPR-Cas9 gene editing technology comes from Kosicki et al. in Nature Biotechnology ${ }^{2}$ entitled "Repair of CRISPR-Cas9-induced double-stranded breaks leads to large deletions and complex rearrangements." This study, from Allan Bradley's group at the Wellcome Sanger Institute, is different because it relies on a robust methodology. The authors

*Clustered Regularly Interspaced Short Palindromic Repeats. employed long-read sequencing to characterize the outcome of CRISPR-Cas9 activity systematically over larger genomic regions than commonly interrogated. They showed that expression of the CRISPR-Cas9 system generated deletions larger than expected, as well as chromosomal rearrangements. These findings were observed in several in vitro models: mouse embryonic stem cells, mouse haematopoietic progenitors expressing Cas9, and an immortalized human retinal epithelial cell line.

At this point, it is unclear how these findings will extrapolate to other contexts, including clinical situations (see below). The Kosicki et al. ${ }^{2}$ study focused on a limited number of targets in a handful of cellular models, and most of the experiments employed sustained expression of CRISPRCas9. Moreover, larger deletions may be easier to detect in pools, as shorter segments are more efficiently amplified by polymerase chain reaction (PCR). Overall, the Wellcome study makes a convincing case that deletions larger than

${ }^{1}$ Mary Lyon Centre and ${ }^{2}$ Mammalian Genetics Unit, Medical Research Council Harwell Institute, Oxfordshire, United Kingdom.

Address correspondence to: Lydia Teboul, MRC Harwell Institute, Harwell Campus, Oxfordshire, OX11 ORD, United Kingdom, E-mail: I.teboul@har.mrc.ac.uk or Andy Greenfield, MRC Harwell Institute, Harwell Campus, Oxfordshire, OX11 ORD, United Kingdom, E-mail: a.greenfield@har.mrc.ac.uk

(c) Lydia Teboul and Andy Greenfield 2018; Published by Mary Ann Liebert, Inc. This Open Access article is distributed under the terms of the Creative Commons License (http://creativecommons.org/licenses/by/4.0), which permits unrestricted use, distribution, and reproduction in any medium, provided the original work is properly cited. 
the immediate segment defined by the sites recognized by the sgRNAs, and other allele rearrangements, can occur. The larger deletions extended over a few kilobases, and their frequency (when evaluated) was unexpectedly high ( $>20 \%$ in some cases). These results are all the more convincing because they echo previous observations with one or several sgRNAs in rodent embryos: others have reported larger than expected deletions on-target and/or complex allele rearrangements, ${ }^{3-5}$ including previous work by the Bradley laboratory. ${ }^{6}$

\section{"Pathogenic Consequences"?}

According to Kosicki et al., these observations may toll the bell for CRISPR-Cas9 as a therapeutic tool:

"The observed genomic damage in mitotically active cells caused by CRISPR-Cas9 editing may have pathogenic consequences." They contend that induced translocations, deletions, or inversions may have longrange effects, altering adjacent loci, possibly leading to a carcinogenic "hit" in stem cells and progenitors, which might become neoplastic over time.

Whether we heed the authors' stark warnings, there is no doubt that the CRISPR-Cas9 methodology is still in its infancy, and many important questions remain. Are the observed genetic rearrangements more acute in selected cellular models? Are mouse embryonic stem cells and cell lines in general unusual in their way of coping with genetic lesions? What is the relevance of those cellular models to other somatic cells with respect to the DNA repair machinery and mitotic activity?

Most of the worrisome rearrangements detected by Kosicki et al. were evidenced after sustained CRISPRCas9 activity and, in some instances, amplification from a pool of clones by PCR. It would be interesting to measure the actual frequency of such events in relevant cell types after exposure to CRISPR-Cas9 conditions that mimic clinical applications. Also, larger but focalized deletions represent a different risk to more complex chromosomal rearrangements. Thus, the occurrence of these different event types should be evaluated individually.

Lastly, if larger-but still localized-rearrangements occur, they represent a risk that needs to be evaluated for each locus on a case-by-case basis, as they will likely depend on genetic context. Ultimately, can we predict these rearrangements? After all, we are not considering a CRISPR-specific issue but rather a feature of DNA repair in eukaryotic cells. More work is needed to understand better and perhaps direct DNA repair in a range of clinically relevant cell types before general conclusions can be drawn.

Looking further ahead, it is clear that more research is required to investigate how various parameters impact CRISPR-Cas9-mediated mutagenesis: cell context, genetic background, delivery method, choice of endonuclease, the gRNAs (sequence, positioning), and-when homology-directed repair is attempted-the nature of the donor template (not employed by Kosicki et al.) to name a few. Additionally, it is likely that there will be renewed efforts to improve screening methods, as screening of products will allow selection of cells with the desired events for tissue engineering. Of course, in some proposed clinical contexts, selection will not be possible. This may result in greater exploration of genome editing methodologies that do not employ DNA cutting, such as base editing. ${ }^{7}$

Careful examination of "on-target" risks of CRISPR-Cas9 genome editing must continue, including in animal models, where in vivo delivery is required and selection is not possible. There will also be renewed discussion of safety and efficacy in the context of possible reproductive uses of human genome editing, ${ }^{8}$ and rightly so.

\section{References}

1. Pruett-Miller SM. It's CRISPR clear: off-target study misses the mark. CRISPR J 2018;1:130-131. DOI: 10.1089/crispr.2018.29013.mil.

2. Kosicki M, Tomberg K, Bradley A. Repair of double-strand breaks induced by CRISPR-Cas9 leads to large deletions and complex rearrangements. Nat Biotech 2018 Jul 16 [Epub ahead of print]; DOI: 10.1038/ nbt.4192.

3. Birling MC, Schaeffer L, André $P$, et al.. Efficient and rapid generation of large genomic variants in rats and mice using CRISMERE. Sci Rep 2017;7:43331. DOI: 10.1038/srep43331.

4. Codner GF, Mianné J, Caulder A, et al. Application of long single-stranded DNA donors in genome editing: generation and validation of mouse mutants. BMC Biol 2018;16:70. DOI: 10.1186/s12915-018-0530-7.

5. Shin HY, Wang C, Lee HK, et al. CRISPR/Cas9 targeting events cause complex deletions and insertions at 17 sites in the mouse genome. Nat Commun 2017;8:15464. DOI: 10.1038/ncomms15464.

6. Boroviak K, Doe B, Banerjee R, et al. Chromosome engineering in zygotes with CRISPR/Cas9. Genesis 2016;54:78-85. DOI: 10.1002/ dvg.22915.

7. Komor AC, Kim YB, Packer MS, et al. Programmable editing of a target base in genomic DNA without double-stranded DNA cleavage. Nature 2016;533:420-424. DOI: 10.1038/nature17946.

8. Greenfield A. Carry on editing. Br Med Bull 2018;1-9. DOI: 10.1093/bmb/ Idy020. 